preprint LA-UR-07-3496

\title{
Complexified Dynamical Systems
}

\author{
Carl M Bender $¥$, Darryl D Holm ${ }^{\dagger}$, and Daniel W Hook ${ }^{\ddagger}$ \\ *Center for Nonlinear Studies, Los Alamos National Laboratory, Los Alamos, NM \\ 87545, USA \\ email: cmb@wustl.edu \\ ${ }^{\dagger}$ Department of Mathematics, Imperial College, London SW7 2AZ, UK \\ and \\ Computer and Computational Science, Los Alamos National Laboratory, \\ MS D413 Los Alamos, NM 87545, USA \\ email: dholm@ic.ac.uk \\ ${ }^{\ddagger}$ Blackett Laboratory, Imperial College, London SW7 2BZ, UK \\ email: d.hook@imperial.ac.uk
}

\begin{abstract}
.
Many dynamical systems, such as the Lotka-Volterra predator-prey model and the Euler equations for the free rotation of a rigid body, are $\mathcal{P} \mathcal{T}$ symmetric. The standard and well-known real solutions to such dynamical systems constitute an infinitessimal subclass of the full set of complex solutions. This paper examines a subset of the complex solutions that contains the real solutions, namely, those having $\mathcal{P} \mathcal{T}$ symmetry. The condition of $\mathcal{P} \mathcal{T}$ symmetry selects out complex solutions that are periodic.

PACS numbers: $05.45 .-\mathrm{a}, 45.20 . J j, 11.30 . \mathrm{Er}$
\end{abstract}

Submitted to: J. Phys. A: Math. Gen.

\section{Introductory Description of Classical $\mathcal{P} \mathcal{T}$ Symmetry}

The differential equations that describe many classical dynamical systems are $\mathcal{P} \mathcal{T}$ symmetric; that is, these equations are invariant under combined space and time reflection. The equation for the simple pendulum [1], the Korteweg-de Vries and generalized Korteweg-de Vries equations [2], the Camassa-Holm equation [2, 3], the SineGordon equation [2], the Boussinesq equation [4], and the classical equations [5, 6, 7, 8] associated with some non-Hermitian quantum-mechanical systems [9, 10, 11, 12] are all $\mathcal{P} \mathcal{T}$-symmetric.

In this paper we focus on Euler's differential equations, which govern the free threedimensional rotation of a rigid body about its center of mass. In dimensionless form these equations may be written very simply as

$$
\dot{L}_{1}=L_{2} L_{3}, \quad \dot{L}_{2}=-2 L_{1} L_{3}, \quad \dot{L}_{3}=L_{1} L_{2} .
$$

$\ddagger$ Permanent address: Department of Physics, Washington University, St. Louis MO 63130, USA 
The three-dimensional classical dynamical system described by the Euler equations has been studied in detail, the critical points are known, the real solutions to these equations have been found, and the structure of the phase-space trajectories is well understood [13. However, until now it has not been noticed that Euler's equations, like the classical differential equations mentioned above, are $\mathcal{P} \mathcal{T}$ symmetric. This paper examines the complex $\mathcal{P} \mathcal{T}$-symmetric solutions to this system of equations. It also explores this wider class of solutions for some other PT-symmetric dynamical systems.

We begin by defining what is meant by $\mathcal{P} \mathcal{T}$ symmetry for classical physical systems. We say that a real differential equation that describes classical dynamics is $\mathcal{P} \mathcal{T}$ symmetric if it remains invariant under the combined operations of parity reflection $\mathcal{P}$, which changes the sign of all spatial coordinates $\mathbf{x}$, and time reversal $\mathcal{T}$, which changes the sign of the time coordinate $t$. Furthermore, since the operation of time reversal in quantum mechanics is associated with complex conjugation, we include complex conjugation in the time-reversal operation when the differential equation is complex. Thus, under $\mathcal{P} \mathcal{T}$ reflection we replace the dependent variable $f(\mathbf{x}, t)$ of a differential equation by $f^{*}(-\mathbf{x},-t)$, where ${ }^{*}$ represents complex conjugation. If $f^{*}(-\mathbf{x},-t)$ satisfies the same differential equation as $f(\mathbf{x}, t)$, then the differential equation is $\mathcal{P} \mathcal{T}$ symmetric.

For the special case of Hamiltonian systems of ordinary differential equations, the solutions $\mathbf{x}(t)=\left[x_{1}(t), x_{2}(t), x_{3}(t), \ldots\right]$ and $\mathbf{p}(t)=\left[p_{1}(t), p_{2}(t), p_{3}(t), \ldots\right]$ represent the dynamical coordinate and momentum variables. Under $\mathcal{P} \mathcal{T}$ reflection $\mathbf{x}(t)$ is replaced by $-\mathbf{x}^{*}(-t)$ and $\mathbf{p}(t)$ is replaced by $\mathbf{p}^{*}(-t)$. For the Euler equations (1), the dependent variables $\left[L_{1}(t), L_{2}(t), L_{3}(t)\right]$ represent the angular momentum $\mathrm{n}$ the rotating frame of a rigid body and therefore these variables transform under $\mathcal{P} \mathcal{T}$ reflection like spatial coordinate variables: $\left[L_{1}(t), L_{2}(t), L_{3}(t)\right] \rightarrow\left[-L_{1}^{*}(-t),-L_{2}^{*}(-t),-L_{3}^{*}(-t)\right]$. It is clear from this definition that the Euler equations (1) are $\mathcal{P} \mathcal{T}$ symmetric. (We treat time $t$ as a real parameter; the case of complex time is not considered in this paper.)

A solution $\mathbf{x}(t)$ to a system of dynamical differential equations is a trajectory in coordinate space parameterized by time $t$. The $\mathcal{P} \mathcal{T}$ reflection of this curve, represented by $-\mathbf{x}^{*}(-t)$, is the mirror image of the original curve $\mathbf{x}(t)$ reflected through the imaginary- $x$ axis. A solution to a differential equation may or may not exhibit the symmetries of the differential equation. An easy way to determine if the solution to a $\mathcal{P} \mathcal{T}$-symmetric differential equation is itself $\mathcal{P} \mathcal{T}$ symmetric is to draw the solution curve and to see if the curve is symmetric with respect to the imaginary axis.

Example 1: Complex classical trajectories for the anharmonic oscillator. The one-dimensional classical anharmonic oscillator Hamiltonian $H=\frac{1}{2} p^{2}+x^{4}$ gives Hamilton's equations

$$
\dot{x}=p, \quad \dot{p}=-4 x^{3} .
$$

This dynamical system is $\mathcal{P} \mathcal{T}$ symmetric. There is one integral of the motion (the energy is conserved), and thus the system can be reduced to a single first-order equation:

$$
\frac{1}{2} \dot{x}^{2}+x^{4}=E \text {, }
$$

where the integration constant $E$ may be complex. Note that even though $(2)$ is $\mathcal{P} \mathcal{T}$ symmetric, its first integral (3) need not respect $\mathcal{P} \mathcal{T}$ symmetry. However, requiring that (3) be $\mathcal{P} \mathcal{T}$ symmetric translates into the physical condition that $E$ be real and allows us to interpret $E$ as an energy. 


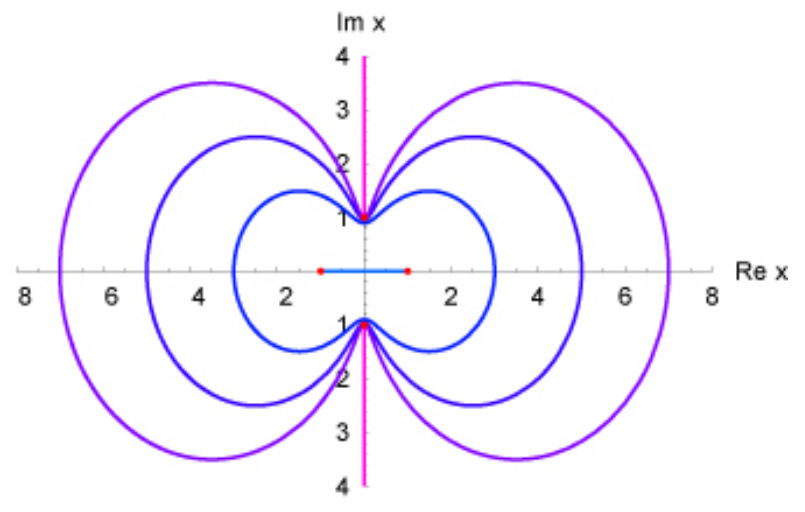

Figure 1. Classical $\mathcal{P} \mathcal{T}$-symmetric trajectories in the complex- $x$ plane representing the possible motions of a particle of energy 1 . This motion is governed by the anharmonic-oscillator Hamiltonian $H=\frac{1}{2} p^{2}+x^{4}$. There is one real trajectory that oscillates between the turning points at $x= \pm 1$ and an infinite family of nested complex trajectories that enclose the real turning points but lie inside the imaginary turning points at $\pm i$. (The turning points are indicated by dots.) Two other trajectories begin at the imaginary turning points and drift off to infinity along the imaginary- $x$ axis. Apart from the trajectories beginning at $\pm i$, all trajectories are closed and periodic. All closed orbits in this figure have the same period $\sqrt{\pi / 2} \Gamma\left(\frac{1}{4}\right) / \Gamma\left(\frac{3}{4}\right)=3.70815 \ldots$.

Suppose first that $E$ is real and positive. We rescale $t$ and $x(t)$ so that $E=1$, and the four turning points in the complex- $x$ plane are located at \pm 1 and $\pm i$. The conventional real periodic motion of the system is represented by a trajectory that lies on the real axis and oscillates between the turning points at \pm 1 (see Fig. 1). However, this real solution to (3) is only one of an infinite number of possible complex trajectories having the same energy. All but two of these trajectories lie outside the real turning points and inside the complex turning points, as shown in Fig. 1. and they are closed and periodic. The remaining two trajectories each begin at the turning points at $\pm i$ and run off to infinity in finite time along the imaginary axis. Note that all of these trajectories are $\mathcal{P} \mathcal{T}$ symmetric; that is, symmetric under reflections about the imaginary axis.

Next, suppose that $E$ is complex. For this case the resulting trajectories are no longer $\mathcal{P} \mathcal{T}$ symmetric and are not closed and periodic. In Fig. 2 the trajectory for a particle whose energy is $E=1+i$ is plotted. The trajectory begins at $x=1$ and does not close.

This anharmonic-oscillator example shows that the conventional real solutions to the classical equations of motion (2) form a trivial lower-dimensional subset of the class of complex $\mathcal{P} \mathcal{T}$-symmetric solutions shown in Fig. 1. The $\mathcal{P} \mathcal{T}$-symmetric solutions in Fig. 1 in turn constitute a subset of the much larger class of all possible complex solutions (see Fig. 2). However, in this paper we limit our attention to the $\mathcal{P} \mathcal{T}$-symmetric classical solutions because these solutions have real energy.

The $\mathcal{P} \mathcal{T}$-symmetric solutions of the classical anharmonic oscillator are special because they form closed and periodic orbits. Indeed, when the classical particles exhibit $\mathcal{P} \mathcal{T}$-symmetric motion in the complex plane, they are bound in a complex classical atom and cannot escape to infinity. (By the term complex atom we mean a localized spacefilling collection of closed and periodic orbits.) If one quantizes this classical system using the Bohr-Sommerfeld quantization condition $\oint d x p=\left(n+\frac{1}{2}\right) \pi$, one obtains the 


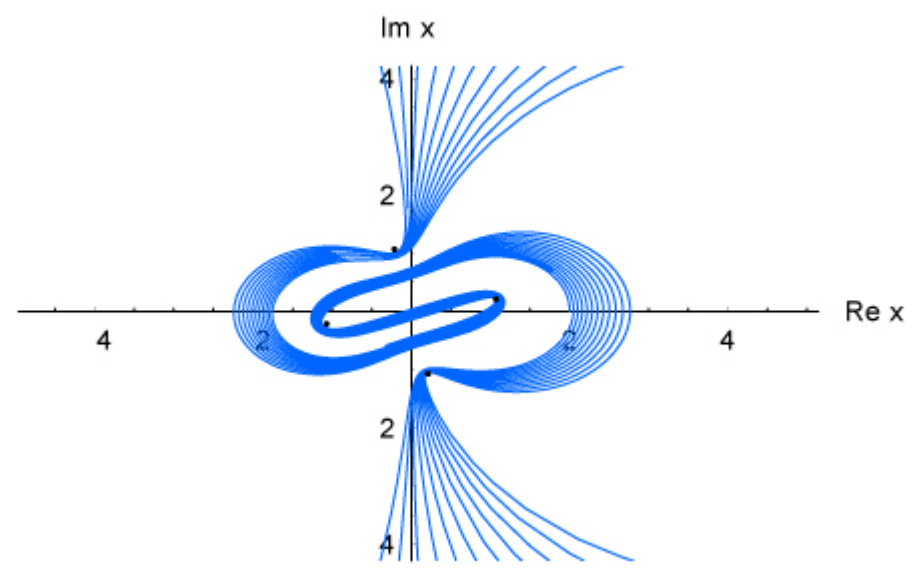

Figure 2. A single non- $\mathcal{P} \mathcal{T}$-symmetric classical trajectory in the complex- $x$ plane for a particle governed by the anharmonic-oscillator Hamiltonian $H=\frac{1}{2} p^{2}+x^{4}$. This trajectory begins at $x=1$ and represents the complex path of a particle whose energy $E=1+i$ is complex. The trajectory is not periodic because it is not closed. The four turning points are indicated by dots.

usual WKB approximation to the discrete energy levels of the quantum anharmonic oscillator, independently of which closed classical orbit is chosen as the integration path. (The Bohr-Sommerfeld quantization condition cannot be applied to the non- $\mathcal{P} \mathcal{T}$ symmetric classical orbit in Fig. 2 because this orbit is not closed and periodic.) Since it is the $\mathcal{P} \mathcal{T}$-symmetric classical orbits that give rise to the energies of the associated quantum system, we regard the $\mathcal{P} \mathcal{T}$-symmetric orbits as physically relevant. Thus, the correspondence principle establishes an association between the family of $\mathcal{P} \mathcal{T}$-symmetric classical orbits and the quantum system.

The Bohr-Sommerfeld quantization condition requires that the classical orbit be closed. Since the trajectory of a particle in the complex- $x$ plane cannot cross itself, the condition of $\mathcal{P} \mathcal{T}$ symmetry is often strong enough to ensure that the trajectory is closed. However, it is possible to have $\mathcal{P} \mathcal{T}$-symmetric trajectories that are not closed [7]. In such cases, the quantum Hamiltonian has complex eigenvalues and is said to have a broken $\mathcal{P} \mathcal{T}$ symmetry. It is possible (though quite rare) to have non- $\mathcal{P} \mathcal{T}$-symmetric trajectories that are closed and periodic, as the following example shows.

Example 2: Closed and periodic trajectories that are not $\mathcal{P} \mathcal{T}$ symmetric. The one-dimensional classical harmonic oscillator Hamiltonian $H=\frac{1}{2} p^{2}+\frac{1}{2} x^{2}$ has complex trajectories that are periodic, but not $\mathcal{P} \mathcal{T}$ symmetric. For this Hamiltonian the equations of motion are

$$
\dot{x}=p, \quad \dot{p}=-x .
$$

These equations are $\mathcal{P} \mathcal{T}$ symmetric. There is one integral of the motion (the energy is conserved), and thus this system can be reduced to the single first-order equation

$$
\dot{x}^{2}+x^{2}=2 E^{2},
$$

where the energy $E^{2}$ is a constant that may be real or complex. The general solution to $(5)$ is $x(t)=E \cos (t+A+i B)$, where $A$ and $B$ are arbitrary real constants. If $E$ is real, then

$$
\operatorname{Re} x(t)=E \cos (t+A) \cosh (B), \quad \operatorname{Im} x(t)=E \sin (t+A) \sinh (B) .
$$




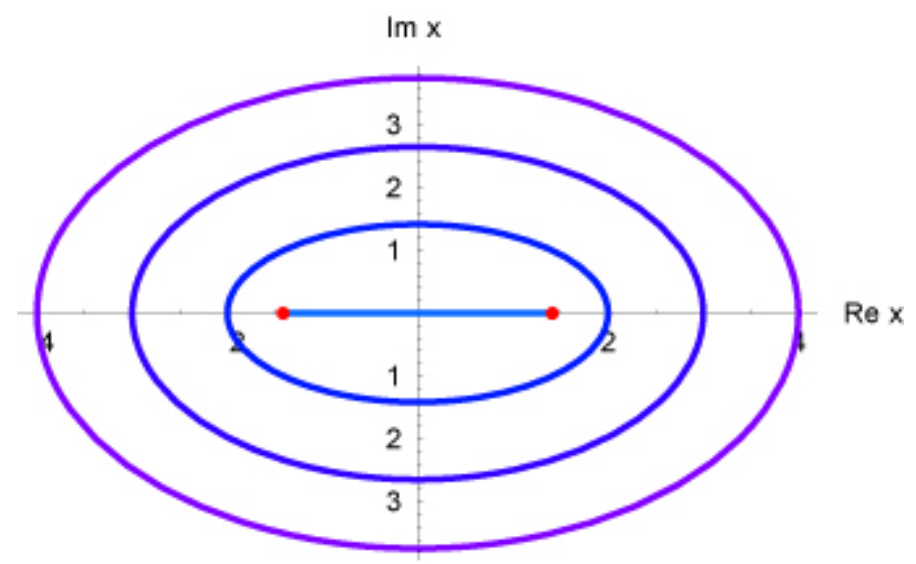

Figure 3. Classical $\mathcal{P} \mathcal{T}$-symmetric trajectories in the complex- $x$ plane for the harmonic-oscillator Hamiltonian $H=\frac{1}{2} p^{2}+\frac{1}{2} x^{2}$. These trajectories are the complex paths $x(t)=E \cos (t+A+i B)$ of a particle whose energy $E^{2}$ is real. The trajectories shown are a family of nested ellipses with foci located at the turning points denoted by dots at $x= \pm E \sqrt{2}$. We have chosen $E$ to be real, so the turning points lie on the real- $x$ axis. (We could equally well have chosen $E$ to be imaginary, and in this case the turning points would lie on the imaginary axis.) The real line segment (a degenerate ellipse) connecting the turning points is the conventional real periodic classical solution to the harmonic oscillator. For $E^{2}=1$ the elliptical trajectories are closed orbits, all having the same period $2 \pi$.

Thus, the graph of the trajectory $x(t)$ in the complex- $x$ plane is the ellipse

$$
[\operatorname{Re} x(t)]^{2}[E \cosh (B)]^{-2}+[\operatorname{Im} x(t)]^{2}[E \sinh (B)]^{-2}=1
$$

with semi-major and semi-minor axes $|E \cosh (B)|$ and $|E \sinh (B)|$. Ellipses of the form in (7) are shown in Fig. 3. These ellipses are $\mathcal{P} \mathcal{T}$ symmetric; that is, they are symmetric with respect to reflections about the imaginary axis. When $E$ is real (imaginary), the turning points at $\pm E$ lie on the real (imaginary) axis. If the energy $E^{2}$ is complex, then the classical trajectories are no longer $\mathcal{P} \mathcal{T}$ symmetric, as we can see in Fig. 4 . However, they are still closed and periodic.

The Lotka-Volterra equations provide a nice two-dimensional example of a $\mathcal{P} \mathcal{T}$ symmetric dynamical system whose complex solutions are generally nonperiodic but whose $\mathcal{P} \mathcal{T}$-symmetric complex solutions are periodic.

Example 3: $\mathcal{P} \mathcal{T}$-symmetric solutions to the Volterra equations: If we generalize slightly the definition of $\mathcal{P}$ reflection to be $\mathcal{P}:(x, y) \rightarrow(y, x)$ [14, then the Lotka-Volterra equations

$$
\dot{x}=x-x y, \quad \dot{y}=-y+x y
$$

become $\mathcal{P} \mathcal{T}$ symmetric. It is well known that the positive real solutions to these equations are periodic they and provide a useful description of predator-prey population dynamics, where $x(t)$ represents the population of the prey species and $y(t)$ represents the population of the predator species. There is one constant of the motion for the Lotka-Volterra equations:

$$
x+y-\log (x y)=C .
$$

For complex solutions $C$ is generally a complex constant, but for $\mathcal{P} \mathcal{T}$-symmetric complex solutions $C$ must be real. 


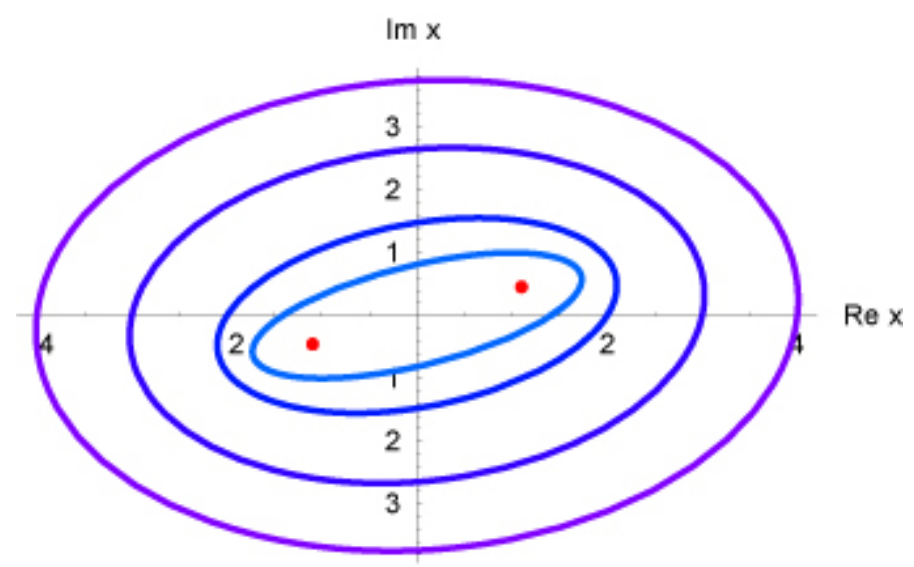

Figure 4. Classical non- $\mathcal{P} \mathcal{T}$-symmetric trajectories in the complex- $x$ plane for the harmonic-oscillator Hamiltonian $H=\frac{1}{2} p^{2}+\frac{1}{2} x^{2}$. These trajectories are complex paths of the form $x(t)=E \cos (t+A+i B)$ for a particle whose energy $E^{2}$ is complex. The trajectories shown are not $\mathcal{P} \mathcal{T}$ symmetric, but they are still closed and periodic. The two turning points are indicated by dots.
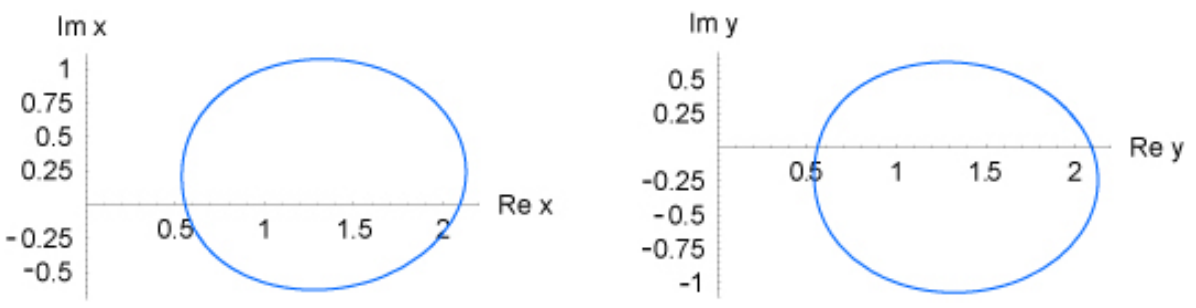

Figure 5. Periodic $\mathcal{P} \mathcal{T}$-symmetric complex solutions to the Lotka-Volterra equations (8). For the initial conditions $x(0)=1+i$ and $y(0)=2.11221-0.403243 i$ the complex trajectories $x(t)$ (left plot) and $y(t)$ (right plot) are shown. Observe that the trajectories are periodic and $\mathcal{P} \mathcal{T}$ symmetric, where $\mathcal{P}$ reflection interchanges $x$ and $y$ and $\mathcal{T}$ reflection consists of complex conjugation.

We first choose the set of initial conditions $x(0)=1+i, y(0)=2.11221-0.403243 i$ for which $C=2$ is real. The complex $\mathcal{P} \mathcal{T}$-symmetric solution, which is plotted in Fig. 5 , is closed and periodic. Next, we choose the set of initial conditions $x(0)=1+i, y(0)=1.09704+1.81173 i$ for which $C=1+i$ is complex. The complex solution, which is plotted in Fig. 6 , is not $\mathcal{P} \mathcal{T}$ symmetric and is not closed and periodic.

Non-Hermitian quantum-mechanical Hamiltonians having unbroken $\mathcal{P} \mathcal{T}$ symmetry are interesting because these operators have only real eigenvalues. Because of the quantum-classical correspondence principle, some light might be shed on the meaning of these quantum states by considering the $\mathcal{P} \mathcal{T}$-symmetric complex solutions of the equations of classical mechanics. The first examples studied have been the $\mathcal{P} \mathcal{T}$ symmetric complex solutions of the one-dimensional nonlinear oscillator $H=p^{2}+x^{2}(i x)^{\epsilon}$ $(\epsilon \geq 0)[9,10,11,12$. The Hermitian quantum version of the Hamiltonian that describes rigid body rotation was treated in the doctoral thesis of Casimir [15]. As far as we know, the $\mathcal{P} \mathcal{T}$-symmetric quantum version of this problem has not yet been treated. 

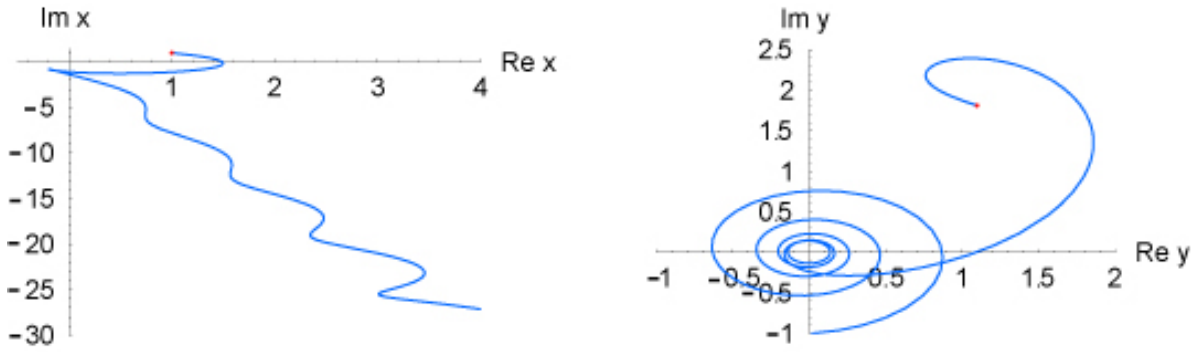

Figure 6. Nonperiodic non- $\mathcal{P} \mathcal{T}$-symmetric complex solutions to the Lotka-Volterra equations (8). For the initial conditions $x(0)=1+i$ and $y(0)=1.09704+1.81173 i$ the complex trajectories $x(t)$ (left plot) and $y(t)$ (right plot) are clearly not periodic and not $\mathcal{P} \mathcal{T}$ symmetric. (The initial conditions are indicated by dots.)

This paper examines the complex $\mathcal{P} \mathcal{T}$-symmetric solutions to Euler's differential equations (1). In Sec. 2 the Euler equations (1) for free rigid-body rotation are derived and their complex solutions are examined. Some concluding remarks are given in Sec. 3 .

\section{Complex Angular Momentum Dynamics}

The complex body-angular-momentum solutions $\mathbf{L}(t) \in \mathbb{C}^{3}$ satisfy Euler's equations [16]

$$
\dot{\mathbf{L}}=\frac{\partial C}{\partial \mathbf{L}} \times \frac{\partial E}{\partial \mathbf{L}}
$$

where $C$ and $E$ are conserved quadratic functions defined by

$$
C(\mathbf{L})=\frac{1}{2} \mathbf{L} \cdot \mathbf{L}, \quad E(\mathbf{L})=\frac{1}{2} \mathbf{L} \cdot \mathbb{I}^{-1} \mathbf{L} .
$$

Here, $\mathbb{I}^{-1}=\operatorname{diag}\left(I_{1}^{-1}, I_{2}^{-1}, I_{3}^{-1}\right)$ is the inverse of the (real) moment-of-inertia tensor in principal-axis coordinates. These equations are $\mathcal{P} \mathcal{T}$ symmetric; they are invariant under spatial reflections of the angular momentum components in the body $P: \mathbf{L} \rightarrow \mathbf{L}$ composed with time reversal $T: \mathbf{L} \rightarrow-\mathbf{L}$.

We now make the simplifying choice $\mathbb{I}^{-1} \equiv \operatorname{diag}(1,2,3)$, which reduces $(10)$ to Euler's dynamical equations in (1). Equation (1) may be written equivalently as

$$
\dot{\mathbf{L}}=\mathbf{L} \times \mathbf{K} \mathbf{L} \quad \text { with } \quad \mathbf{K} \equiv \operatorname{diag}(-1,0,1) .
$$

Since $\mathbf{L}$ is complex, we set $\mathbf{L}=\mathbf{x}+i \mathbf{y}$ and obtain four conservation laws, the real and imaginary parts of $C(\mathbf{L})=\frac{1}{2} \mathbf{L} \cdot \mathbf{L}$ and $H(\mathbf{L})=\frac{1}{2} \mathbf{L} \cdot \mathbf{K} \mathbf{L}=E(\mathbf{L})-2 C(\mathbf{L})$, where

$$
C(\mathbf{L})=\frac{1}{2} \mathbf{x} \cdot \mathbf{x}-\frac{1}{2} \mathbf{y} \cdot \mathbf{y}+i \mathbf{x} \cdot \mathbf{y}, \quad H(\mathbf{L})=\frac{1}{2} \mathbf{x} \cdot \mathbf{K} \mathbf{x}-\frac{1}{2} \mathbf{y} \cdot \mathbf{K} \mathbf{y}+i \mathbf{x} \cdot \mathbf{K} \mathbf{y} .(13)
$$

The solutions to Euler's equations that have been studied in the past are the real solutions to (12), that is, the solutions for which $\mathbf{y}=0$. For this case the phase space is three dimensional and the two conserved quantities are

$$
C=\frac{1}{2}\left(x_{1}^{2}+x_{2}^{2}+x_{3}^{2}\right), \quad H=\frac{1}{2} x_{3}^{2}-\frac{1}{2} x_{1}^{2} .
$$

If we take $C=\frac{1}{2}$, then the phase-space trajectories are constrained to a sphere of radius 1. There are six critical points located at $( \pm 1,0,0),(0, \pm 1,0)$, and $(0,0, \pm 1)$. The 


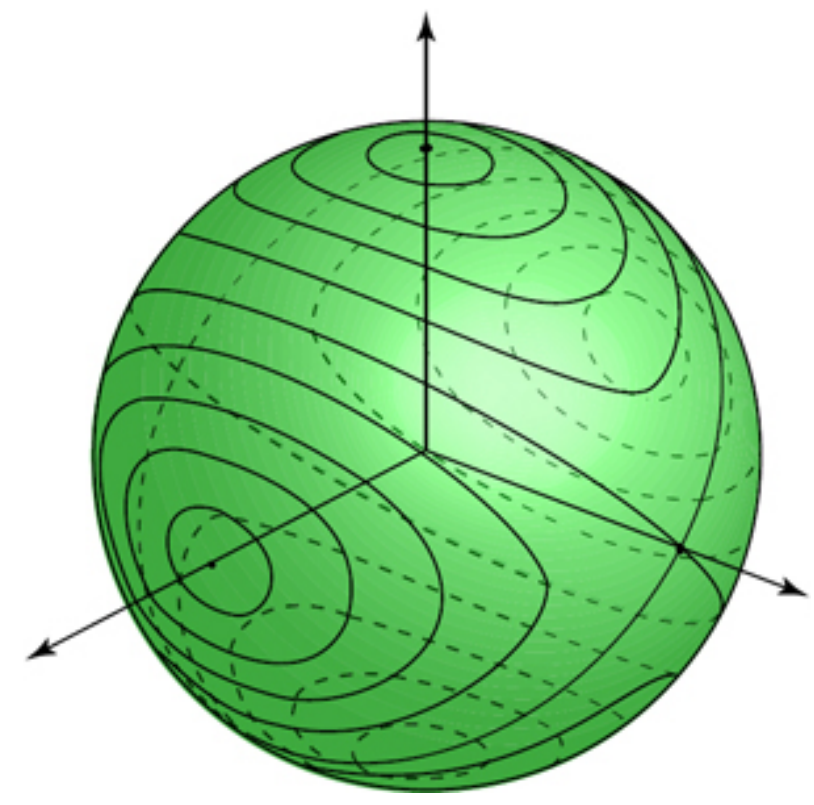

Figure 7. Real phase-space trajectories for Euler's equations (1). Choosing $C=\frac{1}{2}$ in (14) limits these trajectories to the surface of a three-dimensional sphere of radius 1. The critical points lie at $( \pm 1,0,0),(0, \pm 1,0)$, and $(0,0, \pm 1)$. The trajectories for various values of $H$ in 14 are shown.

trajectories for various values of $H$ are shown in Fig. 7. These are the conventional trajectories that are discussed in standard textbooks on dynamical systems [13]. (When $H=0$, the resulting equation is a first integral of the simple pendulum problem [1].)

Let us now examine the complex $\mathcal{P} \mathcal{T}$-symmetric solutions to Euler's equations (12). For this case phase space is six dimensional, which is difficult to visualize. However, the requirement of $\mathcal{P} \mathcal{T}$ symmetry implies that the constants of motion $C$ and $H$ in $(13)$ are real. The vanishing of the imaginary parts of $C$ and $H$ gives the two equations:

$$
\mathbf{x} \cdot \mathbf{y}=0, \quad \mathbf{x} \cdot \mathrm{K} \mathbf{y}=0 .
$$

These two bilinear constraints may be used to eliminate the $\mathbf{y}$ terms in the complex equations (12). When this elimination is performed one obtains the following real equations for $\mathrm{x}$ on the $\mathcal{P} \mathcal{T}$ constraint manifolds (15):

$$
\dot{\mathbf{x}}=\mathbf{x} \times \mathrm{K} \mathbf{x}+M(\mathbf{x}) \mathbf{x}
$$

Here, the scalar function $M=P N / D$, where the functions $P, N$, and $D$ are given by

$$
\begin{aligned}
& P(\mathbf{x})=2 x_{1} x_{2} x_{3}, \quad N(\mathbf{x})=x_{1}^{2}+x_{2}^{2}+x_{3}^{2}-1, \\
& D(\mathbf{x})=\left|\operatorname{Re}\left(\frac{\partial C}{\partial \mathbf{L}} \times \frac{\partial H}{\partial \mathbf{L}}\right)\right|^{2}=x_{1}^{2} x_{2}^{2}+x_{2}^{2} x_{3}^{2}+4 x_{1}^{2} x_{3}^{2} .
\end{aligned}
$$

(This form may be helpful in thinking about the PT-symmetric quantum spin problem.)

The system (16) has nonzero divergence, so it cannot be Hamiltonian even though it arises from constraining a Hamiltonian system. Nonetheless, the system has two 

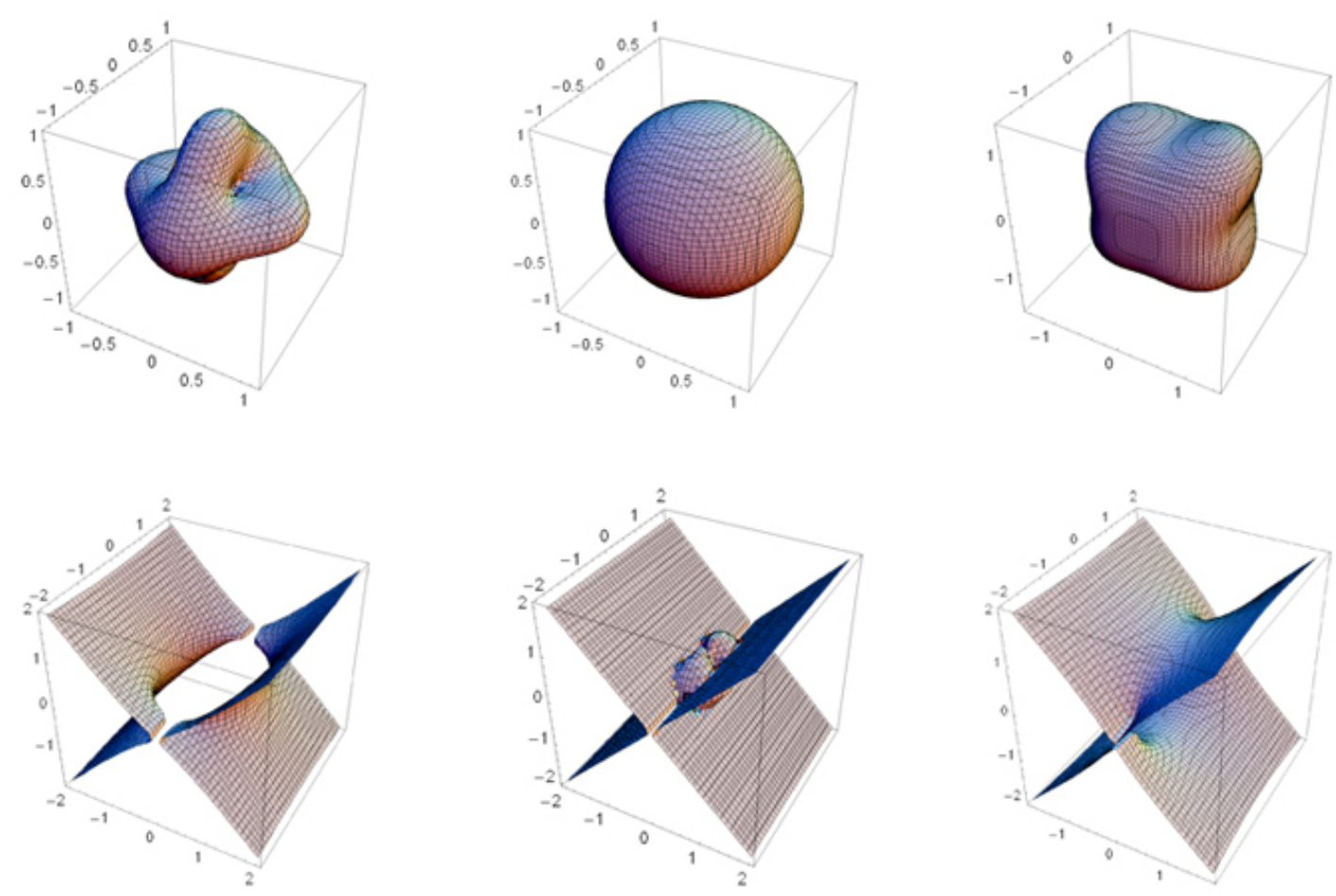

Figure 8. Level surfaces in $\left(x_{1}, x_{2}, x_{3}\right)$ space for the two constants of the motion, $A$ and $B$ in $(19)$. The top row shows the level surfaces corresponding to $A=-1,0$, and 1 and the bottom row shows the level surfaces for $B=-1,0$, and 1 .

additional real conservation laws, and it reduces to the integrable form

$$
\begin{gathered}
\dot{x}_{1}=x_{2} x_{3}\left(1+2 x_{1}^{2} N / D\right), \quad \dot{x}_{2}=-2 x_{1} x_{3}\left(1-x_{2}^{2} N / D\right), \\
\dot{x}_{3}=x_{1} x_{2}\left(1+2 x_{3}^{2} N / D\right)
\end{gathered}
$$

on level sets of two conserved quantities:

$$
A=\frac{(N+1)^{2} N}{D}, \quad B=\frac{x_{1}^{2}-x_{3}^{2}}{D}\left(2 x_{2}^{2} x_{3}^{2}+4 x_{1}^{2} x_{3}^{2}+x_{2}^{4}+2 x_{1}^{2} x_{2}^{2}-x_{2}^{2}\right) .
$$

In Fig. 8 the level surfaces in $\left(x_{1}, x_{2}, x_{3}\right)$ space are displayed for three values of $A$ and for three values of $B$.

The $\mathcal{P} \mathcal{T}$-symmetric trajectories in $\left(x_{1}, x_{2}, x_{3}\right)$ space are characterized by the values of $A$ and $B$ in 19 and these trajectories are precisely the intersections of the level surfaces shown in the top and bottom rows of Fig. 8. For example, in Fig. 9 the level surfaces corresponding to $A=1$ and $B=0$ are superposed. One can see that the intersection of these surfaces is a pair of closed butterfly-shaped curves in $\left(x_{1}, x_{2}, x_{3}\right)$ space. In Fig. 10 many such butterfly-shaped trajectories are shown, and all such trajectories are closed and periodic. All of these trajectories lie outside a unit ball centered at the origin.

In Fig. 11 we display the $\mathcal{P} \mathcal{T}$-symmetric trajectories that lie inside the unit ball centered at the origin. All of these trajectories pass through the origin. However, these 


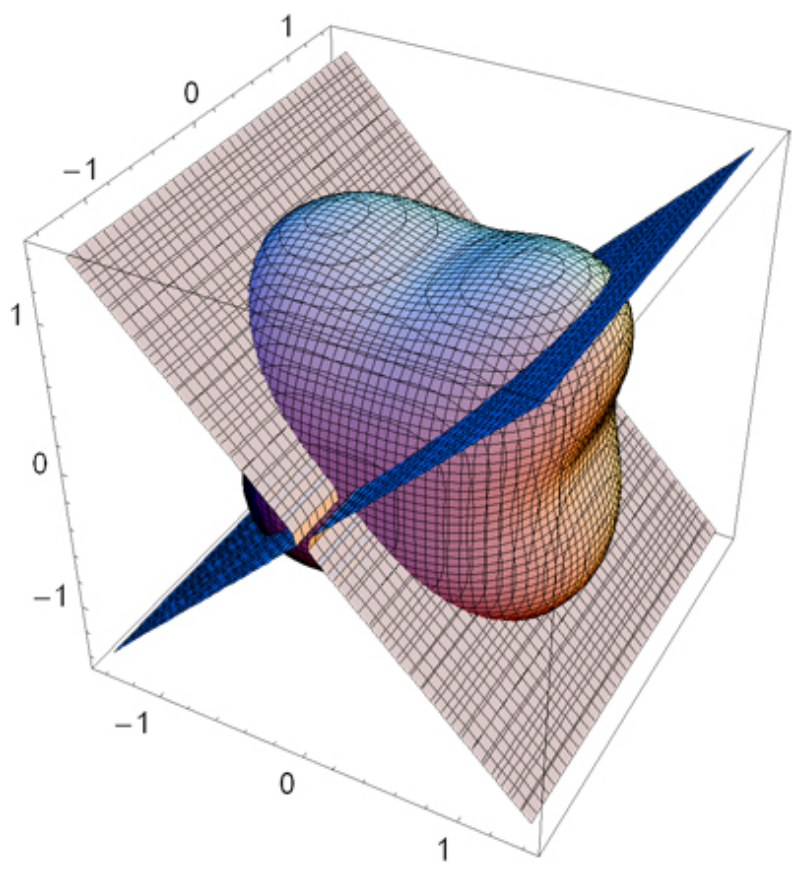

Figure 9. Superposition of two level surfaces in Fig. 8 corresponding to $A=1$ and $B=0$. The trajectory, which is the intersection of these two level surfaces, is a closed butterfly-shaped curve in $\left(x_{1}, x_{2}, x_{3}\right)$ space.

trajectories are periodic and do not stop at the origin. While one can see from (1) that the origin is a critical point for real trajectories, it is clear from (17) and (18) that the origin in $\left(x_{1}, x_{2}, x_{3}\right)$ space is not a critical point.

\section{Conclusions}

In this paper we have used the Euler equations for the free rotation of a rigid body about its center of mass to illustrate the following picture: Given a real dynamical system of differential equations, the real solutions form a tiny subset of the rich and interesting class of complex solutions. If this system is $\mathcal{P} \mathcal{T}$ symmetric, then some of the complex solutions will themselves be $\mathcal{P} \mathcal{T}$ symmetric. The $\mathcal{P} \mathcal{T}$-symmetric solutions are characterized by having real constants of the motion, such as the energy, and thus we view these solutions as being physical. The $\mathcal{P} \mathcal{T}$-symmetric trajectories are different from the other complex trajectories in that they are closed and periodic. For example, if we take the constants of the motion in 13 to be complex, $H=1+i$ and $C=1+i$, then we see in Fig. 12 that while the complex trajectories resemble the butterfly-shaped orbits shown in Fig. 10, they are open rather than closed and periodic orbits. Our results indicate that the following three statements are equivalent: (i) A complex rotating-rigidbody solution is $\mathcal{P} \mathcal{T}$ symmetric; (ii) its constants of motion are real; (iii) it is periodic (except for possible heteroclinic cycles).

It is crucial that the trajectories of a classical dynamical system be multiply 


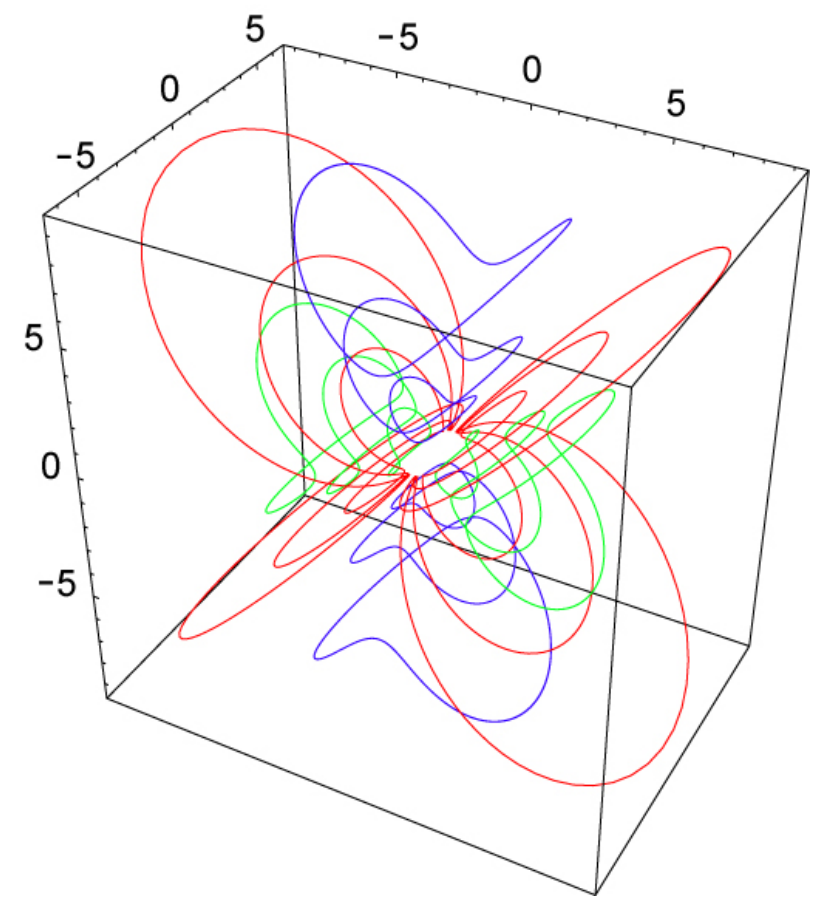

Figure 10. Closed and periodic butterfly-shaped trajectories plotted in $\left(x_{1}, x_{2}, x_{3}\right)$ space. At the center of the figure there are no trajectories indicated. All curves shown in this figure lie outside the unit ball centered at the origin. Trajectories on the unit ball are shown in Fig. 7. The trajectories that lie inside the unit ball are shown in Fig. 11. Trajectories outside the unit ball never cross to the interior of the unit ball.

periodic because only then do the methods of Bohr-Sommerfeld quantization apply. In conventional quantum mechanics only the real phase-space trajectories are considered, and these trajectories terminate at the turning points. On the other side of the turning points is the so-called "classically-forbidden region." We take a broader view and argue that the set of all $\mathcal{P} \mathcal{T}$-symmetric classical orbits constitutes a complex atom. Any one of these orbits can be used to determine the energy levels of the associated quantum system because these orbits are closed and thus Cauchy's theorem implies that the energy levels so obtained will be unique. Furthermore, the reality of the energy levels, which is a crucial property of a physical quantum system, is a consequence of the $\mathcal{P} \mathcal{T}$ symmetry of the underlying classical system.

We thank J. Bender for pointing out Ref. 14] and DWH thanks M. Dixon for helpful conversations. CMB thanks the Mathematics Department at Imperial College, London, for its hospitality. As an Ulam Scholar, CMB receives financial support from the Center for Nonlinear Studies at the Los Alamos National Laboratory. CMB is also supported by a grant from the U.S. Department of Energy. The work of DDH is supported by the Royal Society of London and by the U.S. Department of Energy Office of Science Applied Mathematical Research.

[1] C. M. Bender, D. D. Holm, and D. W. Hook, J. Phys. A: Math. Theor. 40, F81 (2007). 


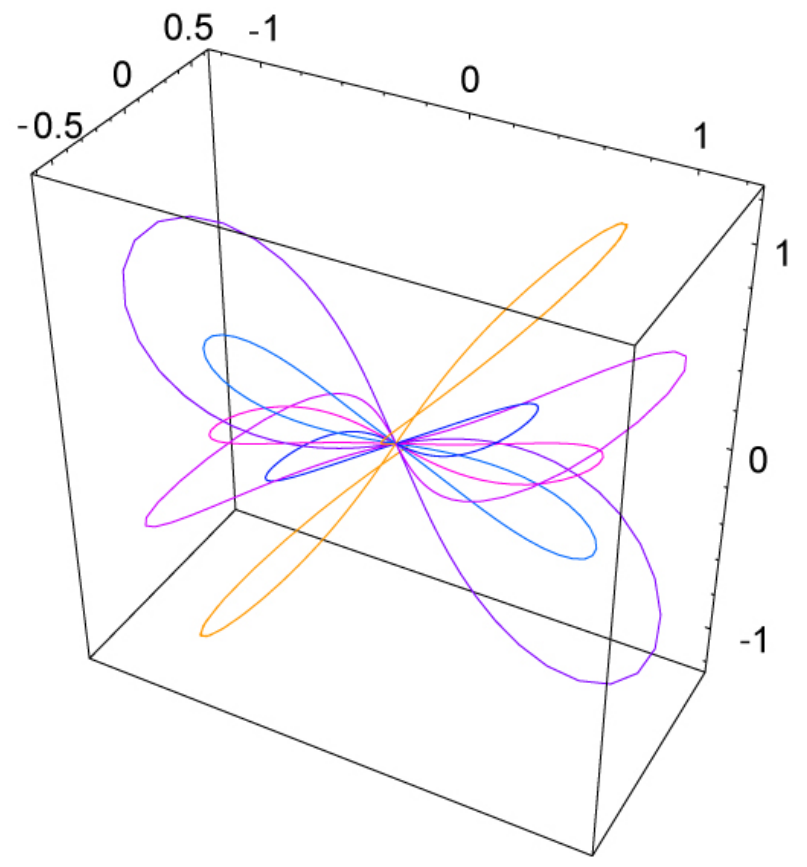

Figure 11. Closed and periodic butterfly-shaped trajectories that lie inside the unit ball centered at the origin. Every trajectory passes through the origin.

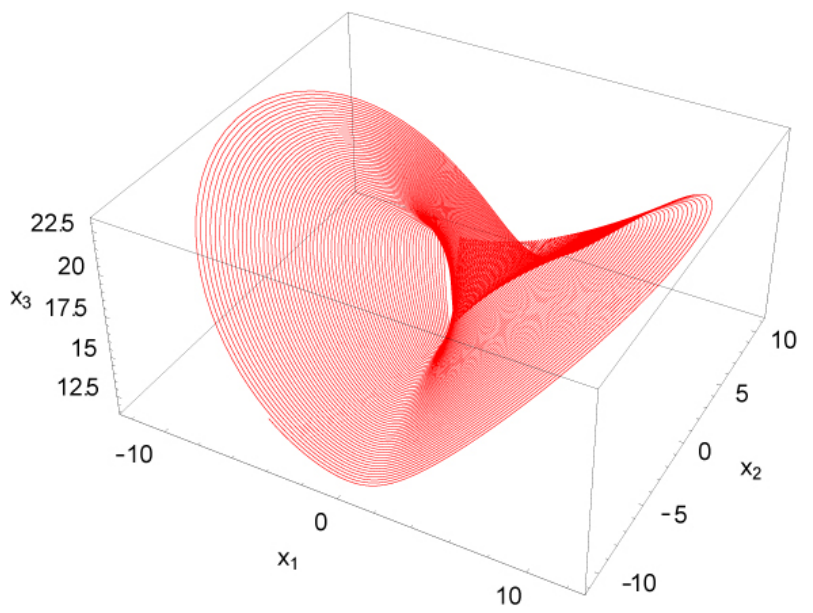

Figure 12. A single nonperiodic butterfly-shaped open trajectory that arises when the constants of motion $H=1+i$ and $C=1+i$ in $(13)$ are complex. The trajectory is not $\mathcal{P} \mathcal{T}$ symmetric and not closed. Rather, it spirals out to infinity.

[2] C. M. Bender, D. C. Brody, J.-H. Chen, and E. Furlan, J. Phys. A: Math. Theor. 40, F153 (2007).

[3] R. Camassa and D. D. Holm, Phys. Rev. Lett 71, 1661 (1993).

[4] G. B. Whitham, Linear and Nonlinear Waves (Wiley-Interscience, New York, 1974).

[5] C. M. Bender, S. Boettcher, and P. N. Meisinger, J. Math. Phys. 40, 2201 (1999).

[6] A. Nanayakkara, Czech. J. Phys. 54, 101 (2004) and J. Phys. A: Math. Gen. 37, 4321 (2004).

[7] C. M. Bender, J.-H. Chen, D. W. Darg, and K. A. Milton, J. Phys. A: Math. Gen. 39, 4219-4238 (2006).

[8] C. M. Bender and D. W. Darg, J. Math. Phys. (to be published) arXiv:hep-th/0703072. 
[9] C. M. Bender and S. Boettcher, Phys. Rev. Lett. 80, 5243 (1998).

[10] C. M. Bender, D. C. Brody, and H. F. Jones, Phys. Rev. Lett. 89, 270401 (2002); Am. J. Phys. 71, 1095 (2003); Phys. Rev. Lett. 93, 251601 (2004).

[11] C. M. Bender, Contemp. Phys. 46, 277 (2005) and arXiv: hep-th/0703096

[12] P. Dorey, C. Dunning, and R. Tateo, J. Phys. A: Math. Gen. 34, L391 (2001); ibid. 34, 5679 (2001); arXiv: hep-th/0703066.

[13] See, for example, J. E. Marsden and T. S. Ratiu, Introduction to Mechanics and Symmetry (Springer-Verlag, New York, 1994) or C. M. Bender and S. A. Orszag, Advanced Mathematical Methods for Scientists and Engineers (McGraw-Hill, New York, 1978).

[14] V. Panković, R. Glavatović, and M. Predojević, arXiv: q-bio/0510020.

[15] H. G. B. Casimir, Z. Phys. 59, 623 (1930).

[16] D. D. Holm and J. E. Marsden, in Symplectic Geometry and Mathematical Physics, P. Donato, C. Duval, J. Elhadad, G. M. Tuynman, ed., Prog. Math. 99 (Birkhauser, Boston, 1991), p. 189. 